\title{
COMPOSICIÓN DEL FITOPLANCTON EN EL ESTRECHO DE BRANSFIELD E ISLA ELEFANTE DURANTE EL VERANO AUSTRAL DE 1999
}

\author{
COMPOSITION OF PHYTOPL.ANKTON IN THE BRANSFIELD STRAIT AND \\ ELEPHANT ISLAND DURING AUSTRAL SUMMER OF 1999
}

\author{
Sonia Sánchez y Patricia Villanueva
}

\section{RESUMEN}

Se da a conocer la composición y distribución de la comunidad fitoplanctónica en los primeros $75 \mathrm{~m}$ de profundidad en el Estrecho de Bransfield y alrededores de la Isla Elefante, durante la Expedición ANTAR X (22-29 enero de 1999) verano austral de 1999. Las mayores concentraciones celulares (>500 $\mathrm{cel} / \mathrm{mL}$ ) estuvieron dadas por el nanoplancton, con núcleos de altas densidades principalmente en las estaciones marginales y en los primeros $25 \mathrm{~m}$ de profundidad. Las especies más representativas fueron Leucocryptos marina, Phaeocystis antarctica, Monadas y las diatomeas Pennatas.

Palabras clave: Fitoplancton antártico, productores primarios, nanoplancton, Verano Austral, Estrecho de Bransfield, Perú ANTAR X.

\section{ABSTRACT}

The authors inform about the composition and distribution of phytoplanktonic community between the first $75 \mathrm{~m}$ of depth in Brarsfield Strait $y$ around the Elephant island, during the ANTAR $X$ expedition in the 1999 Austral Summer (22nd-29th January 1999).

The higher cellular concentration $(500 \mathrm{cel} / \mathrm{mL})$ was given by the autotrophic nanoplankton, with a high density mainly on the bay stations and down the first $25 \mathrm{~m}$ of depth. Among the most representative species we have Leucocryptos marina, Phaeocystis antarctica, the Monadas and the pennate diatoms.

Key words: Antarctic phytoplankton, primary producers, nanoplankton, austral summer, Bransfield Strait, Peru ANTAR $X$.

\section{INTRODUCCIÓN}

La región Antártica ha sido caracterizada en términos de biomasa y producción como uñ ambiente oligotrófico (El-Sayed y Myelli 1965, El-Sayed 1984, Magazzu y Decembrini 1990); sin embargo, es conocida la existencia de áreas altamente productivas con una activa y dinámica cadena alimentaria, condicionada a una muy variable distribución del fitoplancion.

En el Perú, las investigaciones multidisciplinarias en el continente Antártico se iniciaron en $1988 \mathrm{con}$ la primera Expedición Científica ANTAR I. Gómez (1988.

Instituto del Mar del Perú. Lab. Fitoplancton. IMARPE. Aptdo. 22. Chucuito/La Punta. e-mail: soniasan@imarpe.gob.pe
1991), Antonietli, (1989) y Delgado, (1999) reportan cambios marcados en la composición especiológica de la comunidad, caracterizada por una alternancia en la especie dominante dada por las diatomeas o el nanoplancton.

Con el objetivo de mantener la serie histórica en la región Antártica, se da a conocer la composición y distribución especial del fitoplancton entre los primeros $75 \mathrm{~m}$, durante el verano Austral de 1999 (ANTAR X) en el Estrecho de Bransficld e Isla Elefante.

\section{MATERIAL Y'MÉTODOS}

\section{Ârea de Estudio}

El área de esiudio comprende el Estrecho de Bransfield y alrededores de la Isla Elefan- 
te, en los paralelos $62^{\circ}$ y $64^{\circ} \mathrm{S}$, con una extensión máxima de muestreo de $460 \mathrm{~km}$ de largo y $120 \mathrm{~km}$ de ancho entre las Islas Shetland del Sur y Península Antártica (Fig. 1)

\section{Colecta de muestras}

La Décima Expedición Científica Peruana a la Antártica se realizó entre el 22 y 29 de enero de 1999, a bordo del BIC Humboldt, efectuándose un total de 22 estaciones.

Para el análisis cuantitativo se obtuvieron un total de 110 muestras de agua con botella Niskin a las profundidades de $0,10,25,50 \mathrm{y}$ $75 \mathrm{~m}$, obteniéndose en cada una de ellas submuestras de $100 \mathrm{~mL}$.

Para el análisis de la distribución vertical se han considerado 3 perfiles (ests. 1 al 3,7 al 9 y de 13 al 15) localizados en el Estrecho de Bransfield. Todas las muestras fueron preservadas con formalina neutralizada al $1 \%$.

\section{Identificación y recuento celular}

La metodología del tratamiento de las células fue según Balech y Ferrando, (1964). Para el recuento de los organismos se siguió la metodología de Utermohl, (1958) y siguiendo las recomendaciones de UNESCO, (1978). En el laboratorio se procedió a la sedimentación de las muestras en cilindros de $50 \mathrm{~mL}$ $\mathrm{cm}^{3}$ por espacio de 48 horas; los organismos mayores de $20 \mu \mathrm{m}$ fueron contados con un objetivo de $20 x$ y en toda la base, mientras que los flagelados y/o organismos menores de $20 \mu \mathrm{m}$ se contaron con un objetivo de $40 \mathrm{x}$ en un área que representa $2 \mathrm{~cm}^{3}$.

Los organismos fitoplanctónicos fueron identificados hasta especie o el taxa más cercano, expresando los resultados en $\mathrm{N}{ }^{\circ} \mathrm{cel} /$ $\mathrm{mL}$.

\section{Estructura comunitaria}

Los valores de concentración celular del fitoplancton fueron transformados previamente a $\log (x+1)$ para la aproximación de su distribución a la normalidad (Ibáñez y Seguín, 1972).
Fue calculado el índice de diversidad de Shannon y Weaver (1963) siendo que

$H^{\prime}=-S\left[(n i / N)^{*} \log 2(n i / N)\right]$,

donde:

ni = número de individuos de cada especie

$N=$ número total de organismos en la muestra

$\mathrm{H}^{\prime}=$ índice de diversidad

Los resultados se expresan en bits cel ${ }^{-1}$ Así tambiên se calculó el úndice de similaridad de Bray Curils, para la identilicación de las principales asociaciones florísticas. Se elaboró una matriz de similaridad y su posterior clasificación jerárquica acumulativa, usando el método de agruparniento UPGMA. En este análisis sólo fueron empleadas las especies con una frecuencia relativa mayor del $10 \%$ de ocurrencia.

Para el análisis comunitario se utilizó el programa $\triangle$ COM 2 (ver: 2,0), mientras que para la claboración de las cartas de distribución de concentración del fitoplancton se utilizó el programa S.URFER (ver. 6.0).

\section{RESULTADOS}

\section{Composición del fitoplancton}

La comunidad fitoplanctónica durante el verano austral de 1999 se ha caracterizado por la presencia de un total de 111 especies de las cuales 84 fueron diatomeas ( 43 centrales y $4 \mathrm{l}$ pennatas), 22 dinoflagelados, 3 flagelados y 2 silicoflagelados (Tabla 1). Se determinaron algunos organismos del microzooplancton teniendo a los ciliados y tintinidos como sus mejores representantes; entre los primeros destacan los géneros Laboea, Lohmanniella. Strombidium y Tiarina; y entre los segundos Cymatocylis, Epiplocylis, Favella, Salpingella y Tintinnopsis.

Los flagelados autótrofos, donde se tiene agrupado al nanoplancton, fueron los más frecuentes y abundantes dominando en más del 


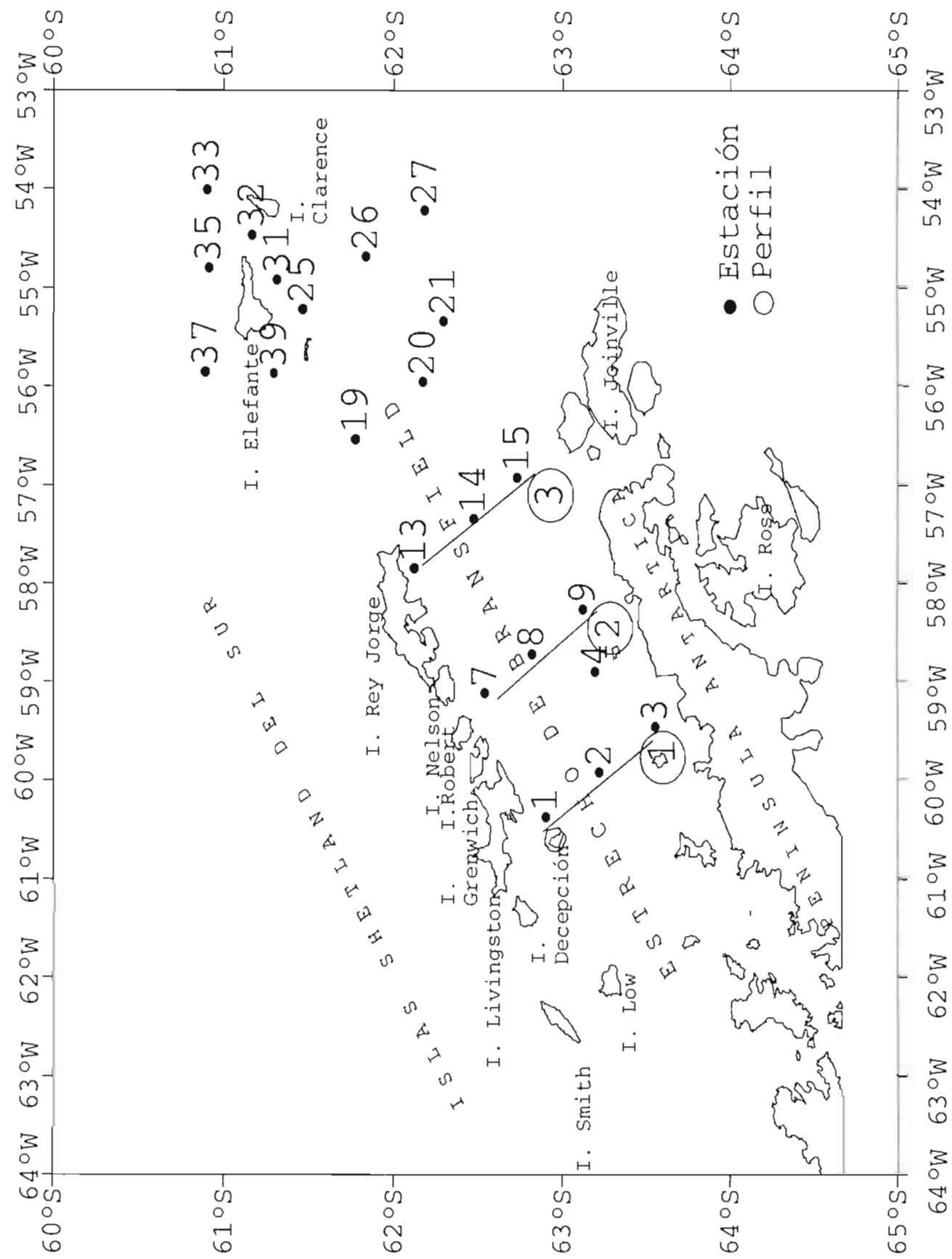

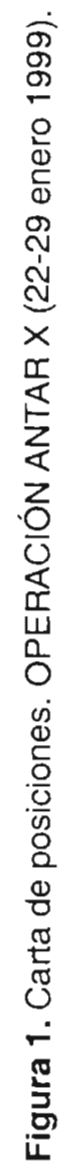




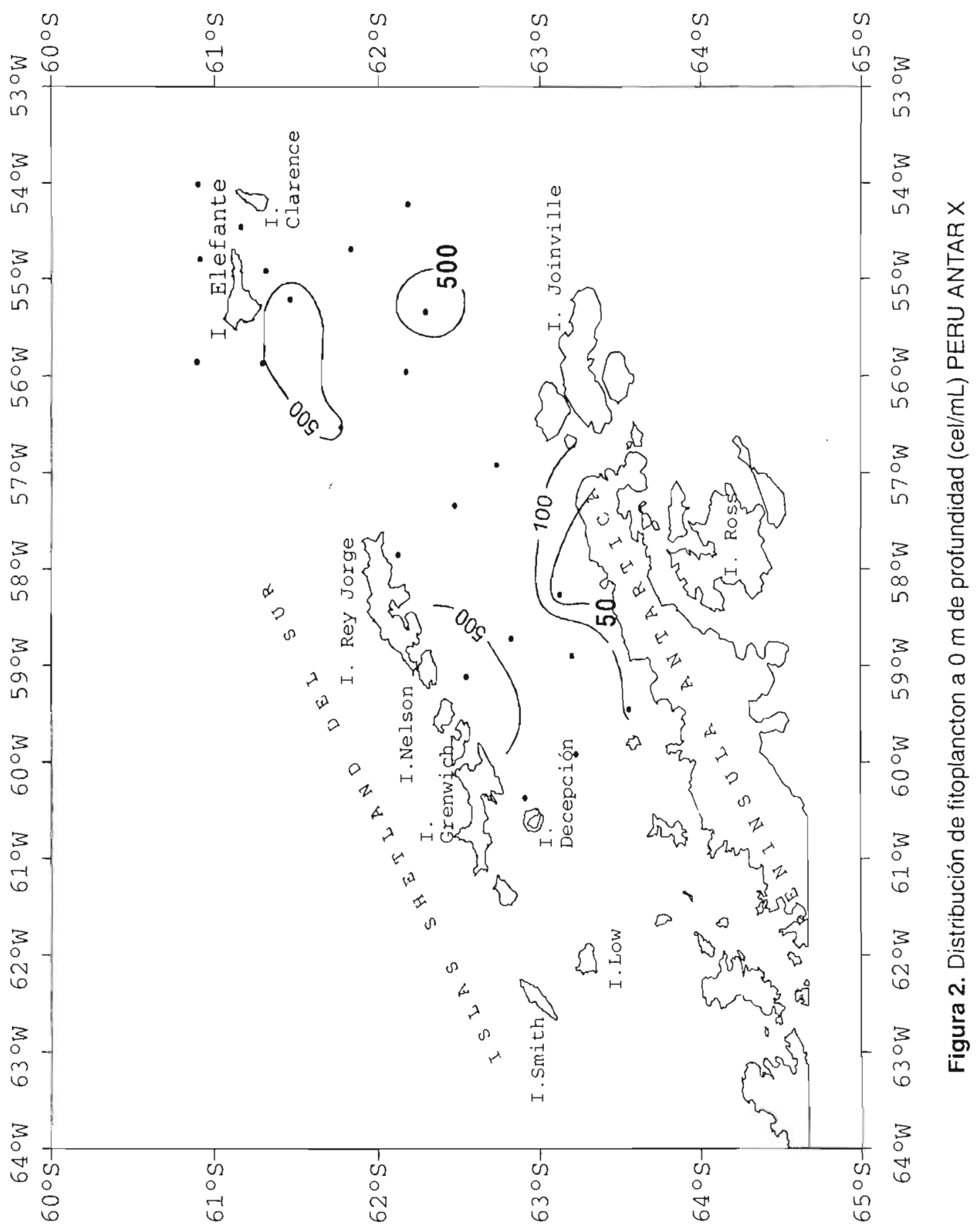




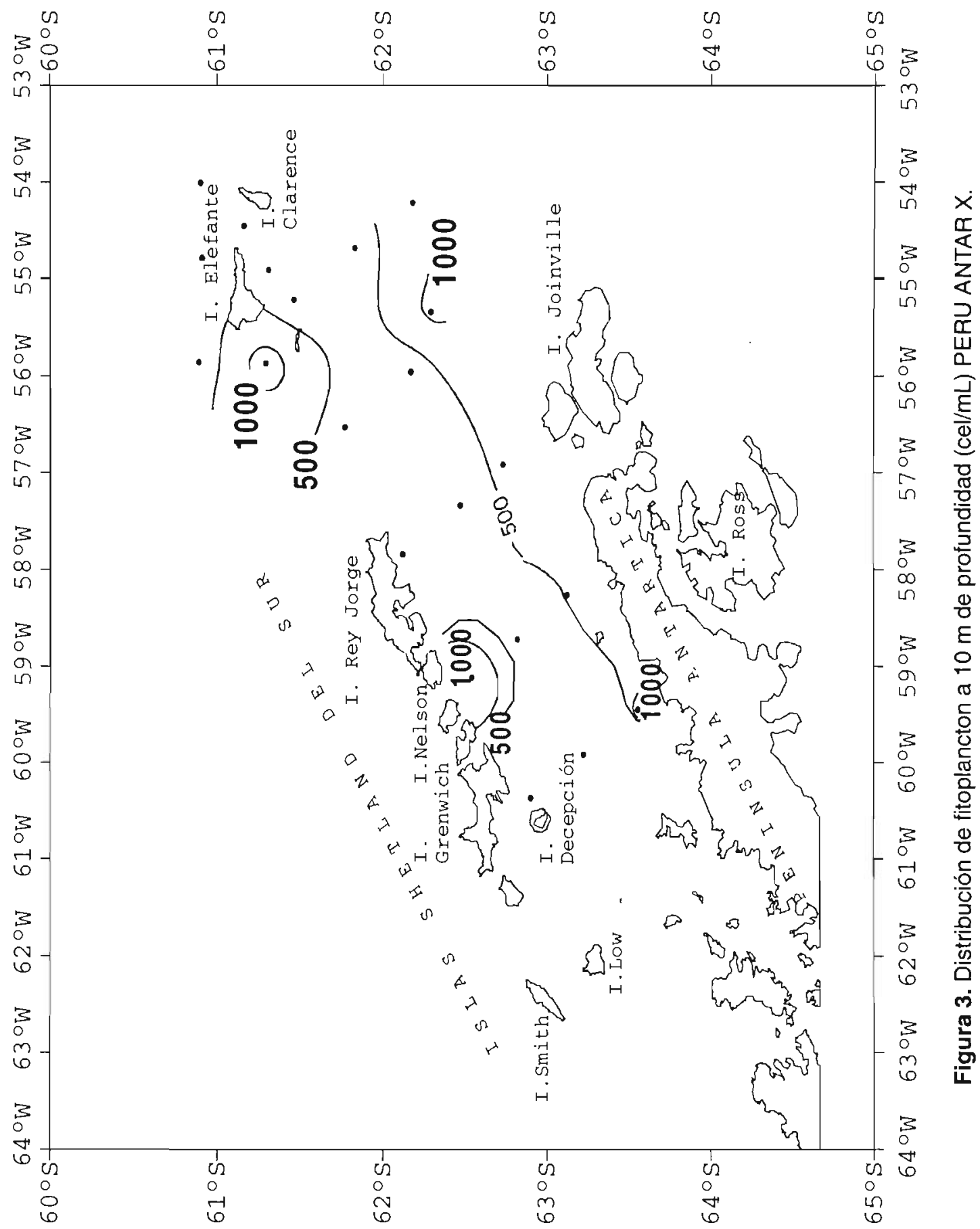


90\% del área estudiada; Leucocryptos marina $(851 \mathrm{cel} / \mathrm{mL})$, Monadas $(720 \mathrm{cel} / \mathrm{mL})$ y Phaeocystis antarctica $(710 \mathrm{cel} / \mathrm{mL})$ fueron las especies más representativas.

Las diatomeas conformaron el segundo grupo en importancia con dominancias menores de $50 \%$, principalmente el grupo de las pennatas con las especies Pseudonitzschia heimii $(113 \mathrm{cel} / \mathrm{mL}), P$. pseudodelicatissima (82 cel/mL) y P. prolongatoides $(76 \mathrm{cel} / \mathrm{mL})$, como las más frecuentes. Las diatomeas centrales mostraron una relativa importancia en número con respecto al primer grupo, alcanzando concentraciones menores de $30 \mathrm{cel} / \mathrm{mL}$. Entre las más frecuentes tenemos a Thalassiosira sp., T. hispida, Corethron criophilum, Chaetoceros neglectum y Actinocyclus cholnokyi.

Los dinoflagelados y silicoflagelados fueron escasos y tuvieron como representantes a Gyrodinium lachryma, Amphidinium hadai, Dissodium gerbaultii y Diplopeltopsis, entre los primeros y entre los segundos a Dictyocha fibula y D. speculum (Tabla 1).

\section{Distribución horizontal y vertical}

En general la concentración celular del fítoplancton fluctuó entre 500 y $1000 \mathrm{cel} / \mathrm{mL}$ (Tabla 2); las mayores concentraciones celulares se localizaron en las estaciones próximas a la línea costera, entre los 10 y 15 m de profundidad, frente a las Islas Shetland del Sur (est. 7), Península Antártica (ests.3, 9, 15 y 2I) y oeste de la Isla Elefante (est. 39), en oposición a las áreas de menor producción localizadas en la parte central del Estrech ‘ de Bransfield (Fig. 3 y 4). En la superfice, las concentraciones celulares fueron menores a 500 , cel/ mL (Fig. 2).

El nanoplancton fue el principal representante que alcanzó valores máximos de 1413 cel/mL (est. 7), seguido del grupo de las diatomeas con concentraciones de $226 \mathrm{cel} / \mathrm{mL}$ (est. 8).
Verticalmente el fitoplancton estuvo distribuido irregularmente; las mayores concentraciones $(1000 \mathrm{cel} / \mathrm{mL})$ se localizaron entre los 10 y $25 \mathrm{~m}$ de profundidad, dentro del Estrecho de Bransfield (perfiles 1 y 2). El perfil 3 muestra una distribución bastante homogénea asociada a densidades menores de $500 \mathrm{cel} /$ $\mathrm{mL}$, con excepción de pequeños "parches" (Fig. 5).

Por debajo de las $30 \mathrm{~m}$ de profundidad puede observarse en general una marcada disminución en las concentraciones totales de fitoplancton que alcanza valores de $50 \mathrm{cel} / \mathrm{mL}$. Las Monadas en todos los casos son las especies más frecuentes y abundantes.

\section{Estructura comunitaria}

El índice de diversidad ( $H^{\prime}$ ) en general fluctuó entre 0,01 y 2,5 bits cel $^{-1}$, mostrando entre los primeros $25 \mathrm{~m}$ de profundidad una distribución bastante homogénea con valores

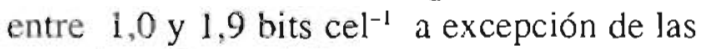
ests. 1 y 13 en el Estrecho de Bransfield y ests. 32 y 31 en la Isla Elefante, en donde los valores superan los 2 bits cel $^{-1}$. Los valores más bajos ( $<1$ bits cel $^{-1}$ ) se registran entre los 50 y $75 \mathrm{~m}$ de profundidad asociados a una baja riqueza de especies (menor de 10, ver Tabla 2).

El análisis de agrupamiento (Fig. 6), al $58 \%$ de similaridad, define 2 grupos de estaciones. El grupo I (GMI) aglomeró principalmente las estaciones del Estrecho de Bransfield, con altas concentraciones ( $>$ $500 \mathrm{cel} / \mathrm{mL}$ ). Entre las especies más frecuentes tenemos a Pseudonitzschia delicatissima, $P$. pseudodelicatissima, $P$. prolongatoides, $P$. heimii, Thalassiosira antarctica, Corethron criophilum, Gyrodinium lachryma y Skeletonema costatum.

El grupo II (GM II), asociado prncipalmente a estaciones con bajas concentraciones $(<500 \mathrm{cel} / \mathrm{ml}$ ) localizadas alrededor de la İsla Elefante y algunas estaciones de la parte central del Estrecho de Bransfield. 


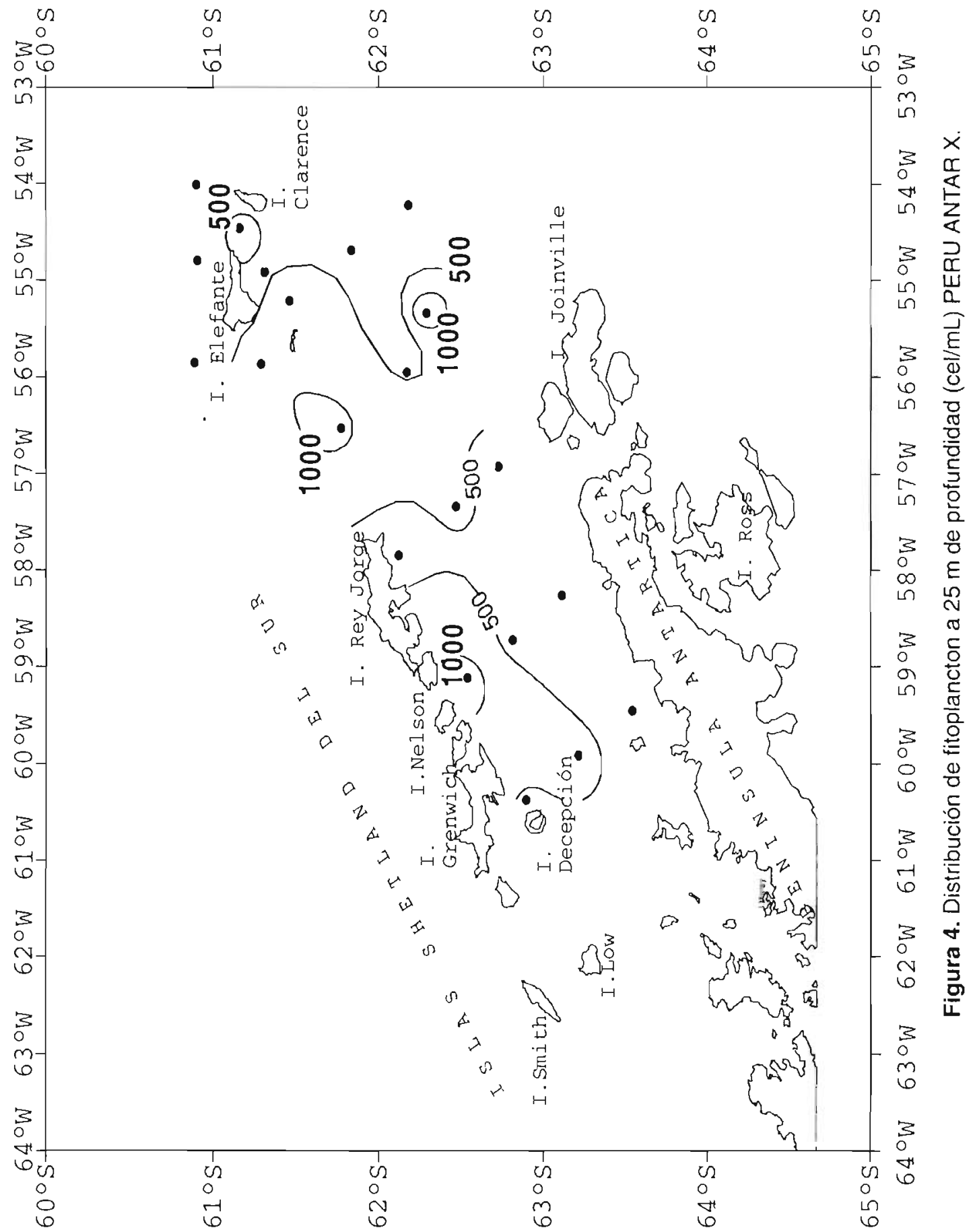




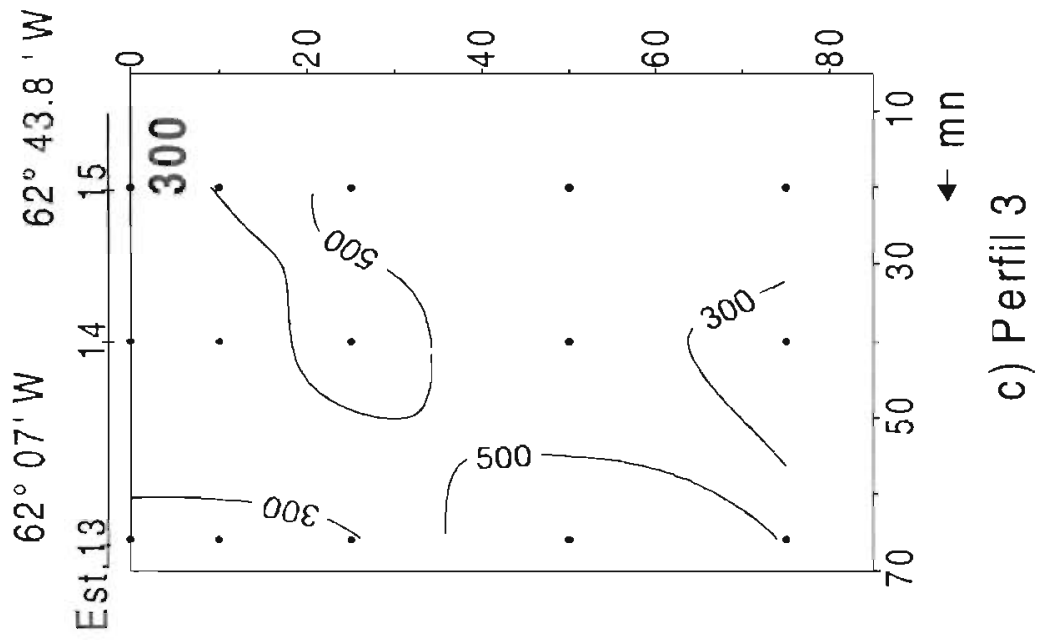

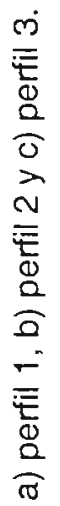
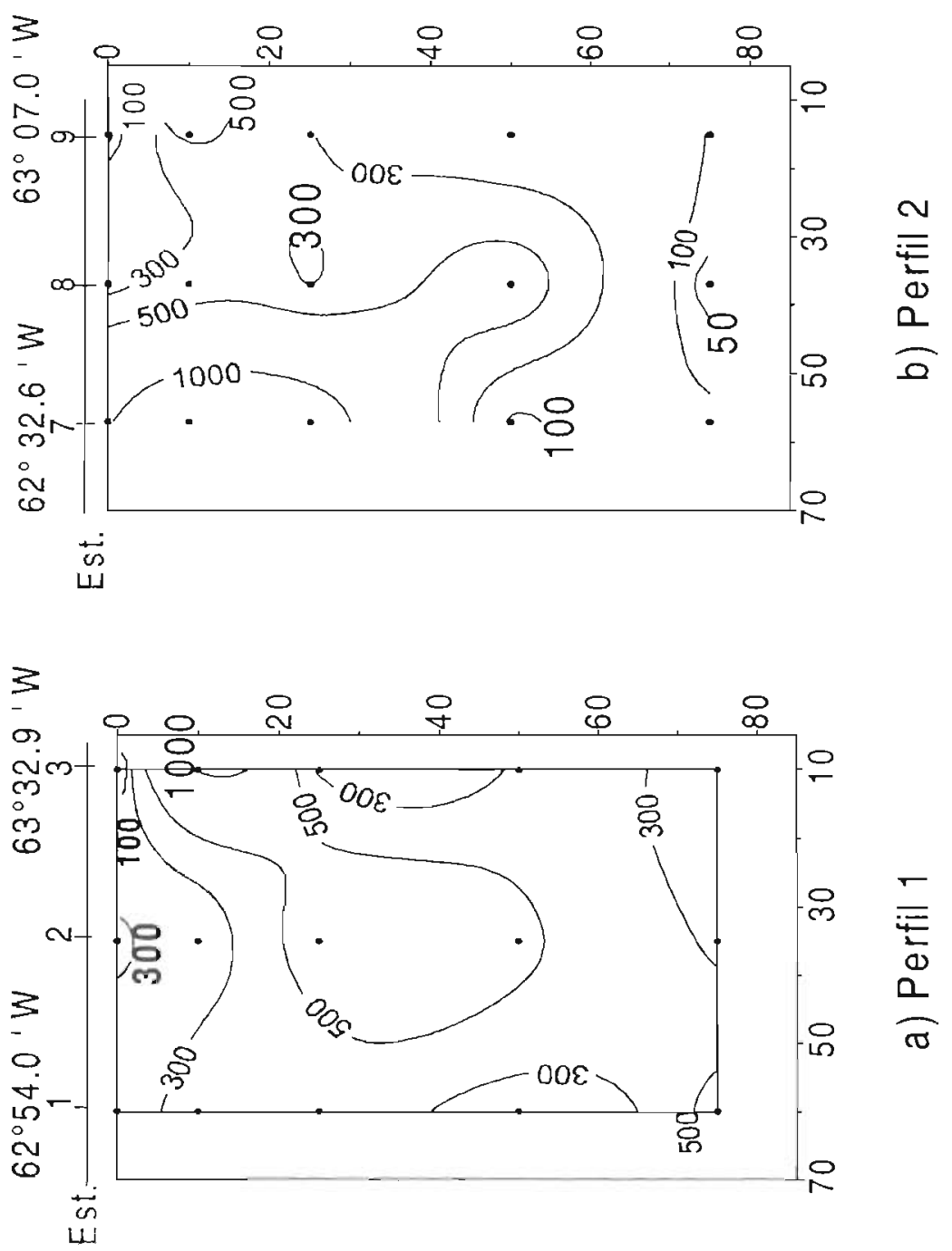
PERU ANTAR X - 1999

$(0 \mathrm{~m})$

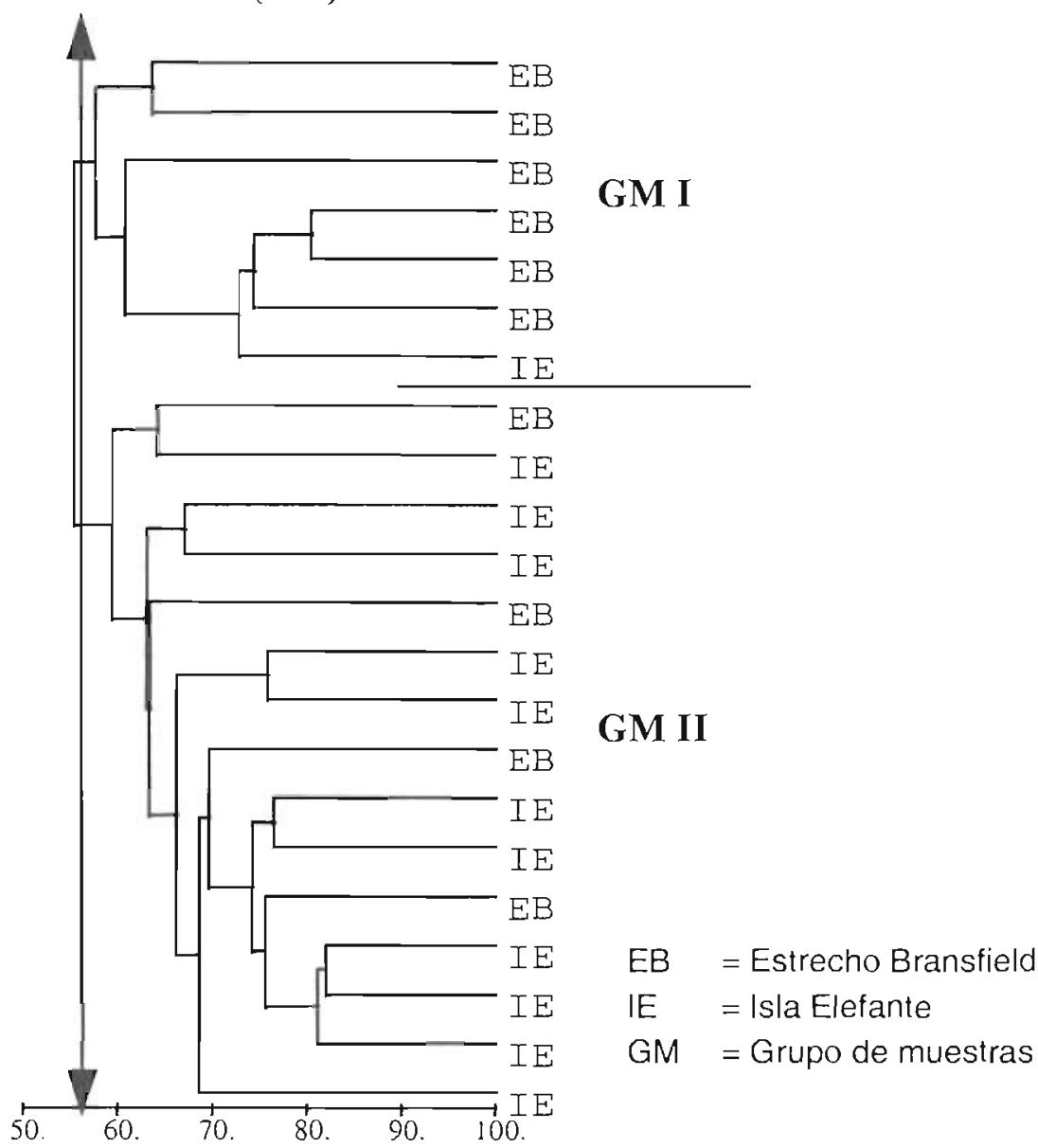

Figura 6. Dendrograma de similaridad (indice Bray-Curtis), BIC Humboldt 9901 (22-29 enero 1999).

Entre las especies más frecuentes tenemos a Pseudonirschia heimii. P. pseudodelicatissima, P. prolongatoides, Phaeocystis antarctica y Thatassiosira hispida (Fig. 6).

\section{DISCUSIÓN}

La composición y distribución del fitoplancton para el verano austral de 1999 en la región Antártica estuvo caracterizada por la frecuencia y abundancia de organismos del nanoplancton, asociados a aguas relativamente más frías. con un contenído alto de oxígeno (Miguel, 2000). Esta comunidad es típica de las primeras fases de la sucesión (Margalef, 1958), como así lo indica el índice de diversi- dad tan bajo en gran parte del área estudiada; características muy similares fueron reportadas por Gómez (1991), Antonietti, (1989) y Delgado (1999), en los veranos respectivos.

La metodología empleada para el conteo de los flagelados ha sido la misma utilizada en otras evaluaciones peruanas en la Antártida y por fines comparativos se mantiene, dando hasta el momento buenos resultados. Los trabajos de Kopczynska, (1992) y Yamamoto \& Taniguchi (1993), entre otros, también utilizan esta metodología, empleando el microscopio de luz para el conteo de los flagelados.

Observaciones de campo para otras expediciones peruanas ANTAR han permitido ob- 
Tabsla 1. Relación de especies del fitoplancton antártico. X Expedición Científica Peruana ANTAR (22-29 enero 1999).

\begin{tabular}{|c|c|}
\hline DIATOMEAS & \\
\hline CENTRALES : & PFNNATAS : \\
\hline Actinocyclus actincrhilus (Ehr.) Simonsen & Pennate $A$ \\
\hline Actinocyclus cholnokyi (Grunow) Van Landingham & Pennate B \\
\hline Actinocyclus curvalulus Janisch & Achnanthes brevipes C. Agardh \\
\hline Actinocyclus sp. & Amphiprora sp. \\
\hline Actinoptinyclus senarius (Ehr.) Ehrenberg & Chuniella antarctica \\
\hline Azpeitia sp. & Chuniella naviculoides \\
\hline Chaetoceros bulbosum (Ehr.) Heiden & Cocconeis imperatrix Schmidt \\
\hline Chaetoceros criophilum Castracane & Cocconeis p. \\
\hline Chaetoceros dichueta Fhrenberg & Cylindrotheca closterium (Ehr.) Reimann \& Lewin \\
\hline Chaetoceros debilis Cleve & Fragilaria sp. \\
\hline Chaetoceros didymus Ehrenberg & Fragilariopsis curta (Van Heurck) Hustedt \\
\hline Chactoceros neglectum Karsien & Fragilariopsis cylindrus (Grunow) Krieger \\
\hline Chaetoceros perwvianum Brightwell & Fragilariopsis kergulelensis ( $\mathrm{O}^{\prime}$ Meara) Hasle \\
\hline Chaetoceros socialis Lauder & Fragilariopsis rilscheri Hustedt \\
\hline Chactoceros sp. & Fragilariopsis rhombica (O'Meara) Hustedt \\
\hline Chaetoceros sp. (esporas) & Fragilariopsis sublinearis Hasle \\
\hline Corethron criophilum Castracane & Grammatophora angulosa Ehrenberg \\
\hline Coscinodiscus asteromphalus Ehrenberg & Grammatophora sp. \\
\hline Coscinodiscus centralis Ehrenberg & Gyrosigma sp. \\
\hline Coscinodiscus concinnus W. Smith & Licmophora gracilis (Ehr.) Giunow \\
\hline Cuscinurdiscus granii Gough & Licmophora sp. \\
\hline Coscinodiscus perforatus Ehrenberg & Manguinea rigida (M. Peragallo) Paddock \\
\hline Coscinodiscus oculoides Ehrenberg & Membraneis challengueri (Grun.) Paddock \\
\hline Coscinodiscus sp. & Navicula distans Medlin \\
\hline Cyclotella sp. & Navicula sp. \\
\hline Dirylum brightwelliii (West) Girunow & Nitzschia longissima Brèbbison \\
\hline Eucampia antarclica (Castr) A52Mangin & Nitzschia promare Medlin \\
\hline Lithodesmium unilulatun Ehrenberg & Nitzschia sp. \\
\hline Odontella weissflogii (Janish) Grunow & Pseudo-nitzschia delicatissima (Cleve) Heiden \\
\hline Porosira glacialis (Gnun) Jorgensen & Pseudo-nitzschia heinuii Mangin \\
\hline Proboscia alaaa (Brightwell) Sundström & Pseudo-nitzschia lineola (Cleve) Hasle \\
\hline Proboscia truncata Karsten & Pseudo-nitzschia prolongatoides Hasle \\
\hline Rluzosolenia rhombus Karsten & Pseudo-ritzschia pseudodelicatissima (Hasle) \\
\hline Rhizosolenia styliformis Brightwell & Psendo-nitzschia pungens (Grunow ex Cleve) \\
\hline Skeletonema costatum (Greville) Cleve & Pscudo-nitzschia seriata (Cleve) H. Peragallo \\
\hline Thalassiosira angulata (Greg.) Hasle & Pseudo-nitzschia sp. \\
\hline Thulassiosira anguste-lineata (A. Schmidt) F. \& Hasle & Pleurosigma sp. \\
\hline Thalassiosira antarctica Comber & Thalassionema frauenfeldii (Grunow) Hallegraeff \\
\hline Thalassiosira bioculata (Gun.) Ostenf. & Thalassionema nitzschioides (Grunow)G. ex Hustedt \\
\hline Thalassiosira gravida Cleve & Thalassiothrix antarctica Schimper (Karsten) \\
\hline Thalassiosira hispida Syvertsen & Trichotoxon reinboldii $\mathrm{H}$. Van Heurck \\
\hline
\end{tabular}

Thalassiosira hyalina (Grun.) Gran

Thalassiosira sp. 
Tabla 1. (continuación)

\section{DINOFLAGELADOS}

Amphidinium hadai Graham

Cercutium furca (Ehr.) Clapurede \& Lachmann

Ceratium fusus ․ fusus (Ehr.) Dujardin

Ceratium rripos (O. F. Muller) Nitzsch

Dinophysis acuminara Claparede \& Lachmann

Dinophysis caudata Saville-Kent

Diplopellopsis sp.

Dissodinium gerbaultii (Pavillard) Taylor

Gonyaulax fusiformis Graham

Gonyaulax sp.

Gymnodinium lohmanni Paulsen

Gymnodinium sp.

Gyrodinium lachryma (Meunier) Kofoid \& Swezy

Gyrodinium sp

Oxyroxum sp.
Prorocentrum gracile Schüt

Prorocentrum micuns Ehrenberg

Proloperidinium conicum (Gran) Balech

Protoperidinium crassipes (Kofoid) Balech

Proloperidinium depressum (Bailey) Balech

Protoperidinium minutum (Kofoid)

Proloperidinium sp.

SILICOFLAGELADOS

Dictrocha fibula Ehrenberg

Dictyocha speculum (Ehrbg.) Lemm.

\section{FLAGELADOS}

Leucocryptos marina (Braarud) Butcher

Monada

Phacocystis ancurctica Karsten servar que, en la mayoría de cilas, las diatomeas centrales están consideradas como el grupo más abundante del fitoplancton; sin embargo; para el verano de 1999, las pennatas logran destacar por su frecuencia y abundancia.

Clarke y Leakey (1996) observaron que los cambios en la flora planctónica esturían asociados principalinente a la formación y tiempo de permanencia de los hielos en la Antártida en donde se puede encontrar una densa población Pseudonitzschias (Fragilariopsis) y Navículas (Schandelmeier y Alexander, 1981).

Se afirma que la región Antártica se carackriza por ser un ambiente oligotrófico (ElSayed y Myelli, 1965; El-Sayed, 1984 y Magazzu y Decembrini 1990). Sin embargo, Nuccio et al. (1992), para un ambiente muy similar catalogaron como áreus de alta productividad a los estratos arriba de los $50 \mathrm{~m}$ de profundidad con concentraciones de $250 \mathrm{cel} /$ mL. Los resultados obtenidos en la presente expedición evidencian una mayor abundancia celular en los primeros $25 \mathrm{~m}$, con concentraciones superiores a $500 \mathrm{cel} / \mathrm{mL}$, principalmente en las zonas mirginales. Córdova y Ledesma (2000) encontraron resultados simi- lares trabajando con datos de clorofila "a".

En cuanto al fitoplancton como alimento disponible, es conocido que el espectro alimentario del Krill (Euphausia superba) consta de una amplia variedad de tamaños de partícula (Miller y Humpton, 1989), sin embargo existiría una preferencia para alimentarse de partículas relativamente grandes como las diatomeas, siendo menos eficiente en atrapar partículas $<20 \mathrm{~mm}$ (Meyer y El-Sayed, 1983). Estudios realizados por Ligowski (1982) y Kopczynska y Ligowski (1982) en áreas con altas concentraciones de Krill, observaron en contenidos estomacales la predominancia de diatomeas en cadenas asociada a una baja densidad de las mismas en las muestras de red.

La distribución bastante homogénea del Krill entre los 10 y $50 \mathrm{~m}$ de profundidad (Escudero y Marín 2001) podría estar condicionando las bajas concentraciones de diatomeas encontradas. favoreciendo las altas concentraciones del nanoplancton. Si bien existen varios factores limitantes en el crecimiento y reproducción del fitoplancton en la Antártida, como incusidad de iuz, incremento del asentamiento de diatomeas con respecto a los flagelados en áreas de mezcla vertical, así 
Tabla 2. Concentración celular de fitoplancton (diatomeas, dinoflagelados, flagelados y fitoplancton total).

\begin{tabular}{|c|c|c|c|c|c|c|c|c|}
\hline Est. & $\begin{array}{l}\text { Lat. } \\
\left({ }^{\circ} \mathrm{S}\right)\end{array}$ & $\begin{array}{l}\text { Long. } \\
\left({ }^{\circ} \mathrm{W}\right)\end{array}$ & $\begin{array}{l}\text { Profund. } \\
(\mathrm{m})\end{array}$ & $\begin{array}{c}\text { Diatom } \\
\left(\mathrm{N} .{ }^{\circ} \mathrm{cel} / \mathrm{mL}\right)\end{array}$ & $\begin{array}{l}\text { Dinoflag. } \\
\left(\mathrm{N} .{ }^{\circ} \mathrm{ce} / \mathrm{mL}\right)\end{array}$ & $\begin{array}{c}\text { Flagelados } \\
\left(\mathrm{N} .{ }^{\circ} \mathrm{cel} / \mathrm{mL}\right)\end{array}$ & $\begin{array}{l}\text { Fitoplancton } \\
\left(\mathrm{N} .{ }^{\circ} \mathrm{cel} / \mathrm{mL}\right)\end{array}$ & $\begin{array}{l}\text { Diversidad } \\
\text { (bits cel }^{-1} \text { ) }\end{array}$ \\
\hline \multirow[t]{5}{*}{1} & $62^{\circ} 54,00$ & $60^{\circ} 23,50$ & 0 & 67 & 0 & 219 & 286 & 1,79 \\
\hline & & & 10 & 77 & 0 & 247 & 324 & 2,07 \\
\hline & & & 25 & 150 & 0 & 280 & 430 & 2,44 \\
\hline & & & 50 & 21 & 0 & 122 & 143 & 1,56 \\
\hline & & & 75 & 7 & 0 & 593 & 600 & 1,38 \\
\hline \multirow[t]{5}{*}{2} & $63^{\circ} 13,10$ & $59^{\circ} 55,80$ & 0 & 3 & 0 & 358 & 360 & 0,87 \\
\hline & & & 10 & 2 & 0 & 211 & 213 & 1,07 \\
\hline & & & 25 & 2 & 0 & 610 & 612 & 1,44 \\
\hline & & & 50 & 1 & 0 & 530 & 530 & 1,08 \\
\hline & & & 75 & 1 & 0 & 262 & 263 & 0,51 \\
\hline \multirow[t]{5}{*}{3} & $63^{\circ} 3,90$ & $59^{\circ} 27,40$ & 0 & 1 & 0 & 76 & 77 & 0,77 \\
\hline & & & 10 & 20 & 0 & 1038 & 1058 & 1,03 \\
\hline & & & 25 & 13 & 0 & 272 & 285 & 1,03 \\
\hline & & & 50 & 2 & 0 & 339 & 341 & 0,54 \\
\hline & & & 75 & 0 & 0 & 119 & 119 & 0,01 \\
\hline 4 & $63^{\circ} 11,71$ & $58^{\circ} 53,86$ & 0 & 1 & 0 & 237 & 238 & 0,25 \\
\hline \multirow[t]{5}{*}{7} & $62^{\circ} 32,60$ & $59^{\circ} 07,00$ & 0 & 1 & 0 & 970 & 971 & 0,93 \\
\hline & & & 10 & 17 & 0 & 1413 & 1429 & 1,06 \\
\hline & & & 25 & 22 & 0 & 1226 & 1248 & 1,08 \\
\hline & & & 50 & 2 & 0 & 63 & 65 & 1.49 \\
\hline & & & 75 & 2 & 0 & 120 & 122 & 0,75 \\
\hline \multirow[t]{5}{*}{8} & $62^{\circ} 49,10$ & $58^{\circ} 43,00$ & 0 & 22 & 0 & 212 & 234 & 1,40 \\
\hline & & & 10 & 131 & 0 & 253 & 384 & 1,99 \\
\hline & & & 25 & 19 & 0 & 267 & 286 & 1,12 \\
\hline & & & 50 & 0 & 0 & 675 & 675 & 0,14 \\
\hline & & & 75 & 0 & 0 & 15 & 15 & 0,18 \\
\hline \multirow[t]{5}{*}{9} & $63^{\circ} 07,00$ & $58^{\circ} 15,00$ & 0 & 1 & 0 & 22 & 23 & 0,93 \\
\hline & & & 10 & 3 & 0 & 499 & 502 & 1,65 \\
\hline & & & 25 & 3 & 0 & 299 & 302 & 1,08 \\
\hline & & & 50 & 31 & 0 & 1.15 & 146 & 1.77 \\
\hline & & & 75 & 1 & 0 & 97 & 98 & 0,61 \\
\hline \multirow[t]{5}{*}{13} & $62^{\circ} 07,16$ & $57^{\circ} 49,43$ & 0 & 21 & 0 & 223 & 245 & 1,05 \\
\hline & & & 10 & 34 & 1 & 203 & 237 & 1,57 \\
\hline & & & 25 & 105 & 0 & 170 & 275 & 2,18 \\
\hline & & & 50 & 58 & 0 & 745 & 803 & 1,19 \\
\hline & & & 75 & 3 & 0 & 468 & 471 & 0,33 \\
\hline \multirow[t]{5}{*}{14} & $62^{\circ} 27,90$ & $57^{\circ} 18,50$ & 0 & 70 & 1 & 325 & 396 & 1,65 \\
\hline & & & 10 & 71 & 1 & 330 & 402 & 1,82 \\
\hline & & & 25 & 44 & 0 & 520 & 564 & 1.87 \\
\hline & & & 50 & 88 & 0 & 240 & 328 & 1,72 \\
\hline & & & 75 & 3 & 0 & 236 & 239 & 1,15 \\
\hline
\end{tabular}


Tabla 2. (continuación).

\begin{tabular}{|c|c|c|c|c|c|c|c|c|}
\hline Est. & $\begin{array}{l}\text { Lat. } \\
\left({ }^{\circ} \mathrm{S}\right)\end{array}$ & $\begin{array}{l}\text { Long. } \\
\left({ }^{\circ} \mathrm{W}\right)\end{array}$ & $\begin{array}{c}\text { Profund. } \\
\text { (m) }\end{array}$ & $\begin{array}{c}\text { Diatom. } \\
\left(\mathrm{N} .^{\circ} \mathrm{cel} / \mathrm{mL}\right)\end{array}$ & $\begin{array}{c}\text { Dinoflag. } \\
\left(\mathrm{N}{ }^{\circ} \mathrm{cel} / \mathrm{mL}\right)\end{array}$ & $\begin{array}{c}\text { Flagelados } \\
\left(N^{\circ} \mathrm{cel} / \mathrm{mL}\right)\end{array}$ & $\begin{array}{l}\text { Fitoplancton } \\
\left.\text { (N. }{ }^{\circ} \text { cel } / \mathrm{mL}\right)\end{array}$ & $\begin{array}{l}\text { Diversidad } \\
{\text { (bits ce }\left.\right|^{-1} \text { ) }}\end{array}$ \\
\hline \multirow[t]{5}{*}{15} & $62^{\circ} 43,80$ & $56^{\circ} 53,00$ & 0 & 2 & 0 & 155 & 157 & 1,11 \\
\hline & & & 10 & 16 & 1 & 516 & 533 & 1,38 \\
\hline & & & 25 & 97 & 0 & 316 & 412 & 1,97 \\
\hline & & & 50 & 3 & 0 & 472 & 475 & 0.85 \\
\hline & & & 75 & 2 & 0 & 471 & 473 & 0,32 \\
\hline \multirow[t]{4}{*}{19} & $61^{\circ} 45,90$ & $56^{\circ} 29,20$ & 0 & 71 & 1 & 444 & 515 & 1,49 \\
\hline & & & 10 & 6 & 0 & 286 & 292 & 1,36 \\
\hline & & & 25 & 131 & 0 & 984 & 1115 & 1,64 \\
\hline & & & 50 & 78 & 0 & 201 & 279 & 1,93 \\
\hline \multirow[t]{5}{*}{20} & $62^{\circ} 10,10$ & $55^{\circ} 54,00$ & 0 & 36 & 0 & 101 & 136 & 1,41 \\
\hline & & & 10 & 20 & 0 & 186 & 206 & 1,55 \\
\hline & & & 25 & 35 & 1 & 360 & 396 & 1,55 \\
\hline & & & 50 & 44 & 0 & 121 & 165 & 1,95 \\
\hline & & & 75 & 0 & 0 & 16 & 16 & 0,42 \\
\hline \multirow[t]{5}{*}{21} & $62^{\circ} 17,30$ & $55^{\circ} 16,40$ & 0 & 1 & 0 & 714 & 715 & 0,83 \\
\hline & & & 10 & ] & 0 & $1] 13$ & $1] 14$ & 0,80 \\
\hline & & & 25 & 1 & 0 & 1175 & 1176 & 1,23 \\
\hline & & & 50 & 0 & 0 & 271 & 271 & 1,02 \\
\hline & & & 75 & 3 & 0 & 246 & 249 & 0,67 \\
\hline \multirow[t]{5}{*}{25} & $61^{\circ} 27,60$ & $55^{\circ} 08,80$ & 0 & 17 & 1 & 582 & 600 & 1,16 \\
\hline & & & 10 & 3 & $L$ & 104 & 108 & 1,68 \\
\hline & & & 25 & 23 & 1 & 532 & 556 & 1,18 \\
\hline & & & 50 & 5 & 0 & 454 & 459 & 0,97 \\
\hline & & & 75 & 4 & 0 & 152 & 157 & 0,94 \\
\hline \multirow{5}{*}{26} & $61^{\circ} 49,60$ & $54^{\circ} 36,60$ & 0 & 85 & 0 & 100 & 185 & 1.70 \\
\hline & & & 10 & 43 & 1 & 152 & 196 & 1,90 \\
\hline & & & 25 & 73 & 0 & 221 & 294 & 1.95 \\
\hline & & & 50 & 104 & 0 & 168 & 272 & 1,86 \\
\hline & & & 75 & 3 & 0 & 242 & 245 & 0,52 \\
\hline \multirow[t]{5}{*}{27} & $62^{\circ} 10,60$ & $54^{\circ} 08,10$ & 0 & 16 & 0 & 185 & 201 & 0,87 \\
\hline & & & 10 & 53 & 0 & 880 & 933 & 1,37 \\
\hline & & & 25 & 2 & 0 & 307 & 309 & 1,45 \\
\hline & & & 50 & 14 & 0 & 305 & 319 & 1.21 \\
\hline & & & 75 & 1 & 0 & 167 & 168 & 0,68 \\
\hline \multirow[t]{5}{*}{31} & $61^{\circ} 18,60$ & $54^{\circ} 50,60$ & 0 & 100 & 13 & 278 & 391 & 1,83 \\
\hline & & & 10 & 66 & 0 & 247 & 313 & 2.06 \\
\hline & & & 25 & 68 & 0 & 394 & 462 & 2.03 \\
\hline & & & 50 & 47 & 0 & 806 & 853 & 1,43 \\
\hline & & & 75 & 7 & 0 & 44 & 51 & 2,13 \\
\hline \multirow[t]{4}{*}{32} & $61^{\circ} 09,60$ & $54^{\circ} 22,90$ & 0 & 183 & 1 & 217 & 400 & 2,22 \\
\hline & & & 10 & 109 & 1 & 34 & 144 & 2,52 \\
\hline & & & 25 & 162 & 1 & 402 & 565 & 1,85 \\
\hline & & & 50 & 6 & 0 & 625 & 631 & 0,58 \\
\hline
\end{tabular}


Tabla 2. (continuación).

\begin{tabular}{|c|c|c|c|c|c|c|c|c|}
\hline Est. & $\begin{array}{l}\text { Lat. } \\
\left({ }^{\circ} \mathrm{S}\right)\end{array}$ & $\begin{array}{l}\text { Long. } \\
\left({ }^{\circ} \mathrm{W}\right)\end{array}$ & $\begin{array}{l}\text { Profund } \\
\text { (m) }\end{array}$ & $\begin{array}{c}\text { Diatom. } \\
\left(\mathrm{N} .{ }^{\circ} \mathrm{cel} / \mathrm{mL}\right)\end{array}$ & $\begin{array}{c}\text { Dinoflag. } \\
\left(\mathrm{N} .{ }^{\circ} \mathrm{cel} / \mathrm{mL}\right)\end{array}$ & $\begin{array}{c}\text { Flagelados } \\
\left(\mathrm{N} .{ }^{\circ} \mathrm{cel} / \mathrm{mL}\right)\end{array}$ & $\begin{array}{c}\text { Firoplancton } \\
\text { tot. }(\mathrm{N} * \mathrm{cel} / \mathrm{mL})\end{array}$ & $\begin{array}{l}\text { Diversidad } \\
\text { (bits cel }^{-1} \text { ) }\end{array}$ \\
\hline \multirow[t]{5}{*}{33} & $60^{\circ} 53.70$ & $53^{\circ} 55,40$ & 0 & 72 & 0 & 115 & 187 & 1.64 \\
\hline & & & 10 & 40 & 0 & 102 & 202 & 1.82 \\
\hline & & & 25 & 16 & 0 & $: 51$ & 167 & 1.01 \\
\hline & & & 50 & 16 & 0 & 348 & 364 & 1,19 \\
\hline & & & 75 & 3 & 0 & 135 & 138 & 0,31 \\
\hline \multirow[t]{5}{*}{35} & $62^{\circ} 54,30$ & $54^{\circ} 43,20$ & 0 & 35 & 0 & 206 & 332 & 1,24 \\
\hline & & & 10 & 91 & 1 & 269 & 361 & 1,87 \\
\hline & & & 25 & 56 & 0 & 296 & 352 & 1,87 \\
\hline & & & 50 & 1 & 0 & 29 & 30 & 1,10 \\
\hline & & & 75 & 1 & 0 & 153 & 154 & 0,39 \\
\hline \multirow[t]{5}{*}{37} & $60^{\circ} 53,50$ & $55^{\circ} 47.90$ & 0 & 99 & 0 & 96 & 195 & 1,92 \\
\hline & & & 10 & 6 & 1 & 162 & 169 & 1,53 \\
\hline & & & 25 & 17 & 0 & 121 & 138 & 1,93 \\
\hline & & & 50 & 49 & 0 & 251 & 301 & 1,89 \\
\hline & & & 75 & 87 & 0 & 182 & 200 & 1,34 \\
\hline \multirow[t]{5}{*}{39} & $61^{\circ} 17,20$ & $55^{\circ} 48,60$ & 0 & 3.3 & 0 & 463 & 496 & 1,21 \\
\hline & & & 10 & 69 & 1 & 1220 & 1290 & 1,25 \\
\hline & & & 25 & 86 & 0 & 904 & 990 & 1,57 \\
\hline & & & 50 & 1 & 0 & 110 & 111 & 1,50 \\
\hline & & & 75 & 1 & 0 & 20) & 21 & 0,57 \\
\hline
\end{tabular}

como la presión de pastoreo ejercido por el Krill, entre otros (Kopczynska, 1992; HolmHansen y Mitchell, 1991), no se puede descartar este último factor como condicionante de la dominancia de los organismos más pequeños.

\section{Agradecimientos}

A la Biologa Flor Chang L. por su participación en los análisis de las muestras, así como a la Blga. Carla Aguilar por su apoyo en la recopilación bibliográfica. Hacemos extensivo nuestro agradecimiento a todas las personas que de alguna otra forma hicieron posible la realización del presente trabajo.

\section{LITERATURA CITADA}

Antonietti. E. 1989. Microplancton del Éstrecho de Bransfield. Verano de 1989 (Pirú ANTAR IJ). Informe Científico de la Segunda Expedición Peruana a la Antítida: 9-44.

Clarke, A. \& R. Leakey. 1996. The seasonal cycle of phytoplinkton, maeronutrients, and the microbial community in a nearshore antarctic marine ecosystem. Limnol. Oceanogr. 41(6): $1281-1294$.
Córdova, J. y J. Ledesma. 2000. Condiciones hidroquimicas en el Estrecho de Bransfield y alredidor de la Isla Elefante durante el verano austral 1999. Informe-sobre las actividades científicas de la Décima Expedición Peruana a la Antártida ANTAR X. Comisión Nacional Asuntos Antárticos. CONCYTEC. Lima Perú: 159-180.

Delgado, E. 1994. Fitoplancion del Estrecho de Bransficld e isla thliante durante el varano austral 1998. Resultadus del programa de Investigación Antártica del Instiuto del mar del Perú Verano Austral 1998. Perú ANTAR TX. Sigunda parte. Crucero BIC Humbold 9801 InI. IMARPE: 4-22.

El-Sayed, S., 1984. Productivity of Antarclic waters. In: Holm-Hansen O., L. Bolis y R. Gilles (eds.). Marine phytoplankion y productivity. Springer-Vrlag. Berlin: 19-34.

El-Sayed, S. \& E. Myelli, 1965. Primary production y stying crop of phytoplankton in the Weddell Sea y Drake Passage. Antarctic Research Serics-American Geophysical Union, Biology of the Antarclic Seas. Vol 5: 87-106.

Éscudero, L. y D. Marín, 2001. Correlación de las variables oceanogáficas en función de la distribución vertical del krill (Euphausia superba) a lo largo del Estrecho de Bransfield. Verano Austral 1999. Inf. 1MAR:PE (160) En prensa.

G6mez. O. 1988. Microplancion del Estrecho de Bransfield durante el verano de 1988. 
Scientific Report of First Peruvian Expedition to Antartida. National Commision of Antartic Affairs (CONAAN): $115-1706$

1991. Microplancton del Estrecho de Bransfield durante el verano de 1991. Informes Científicos de la III Expedición del Perú a la Antartida, Comisión Nacional de Asuntos Antárticos (CONAAN): 109-164.

Holm-Hansen, O. \& B. G. Mitchell, 1991. Spatial and temporal distribution of phytoplankion and primary production in the western Bransfield Strait region. Deep Sea Research. Vol. 38 (89): 961-981.

lbánez, F. et F. Seguín, 1972. Étude du cycle annuel du zooplancton d'Abidjan. Comparaison de plusieurs methodes d'analyses multivariables: composantes principales. Inv. Pesq. 36: 81108.

Ligowski, R. 1982. Phytogenic food of Euphausia superba Dana caught in the southern Drake Pasaje and the Bransfield Strait. FebruaryMarch 1981 (BIOMASS-FIBEX).Pol.Polar Res., 3: 28I-288.

Kopczynska, E. \& R. Ligowski, 1982. Phytoplankton abundance and distribution in the southern Drake Passage and the Bransfield Strait in February-March 1981 (BIOMASS FIBEX). Pol. Polar Res., 3: 193-202.

Kopczynska, E., 1992, Distribution of microflagellates over diatoms in the Antartic areas of deep vertical mixing and krill concentrations. Journal of Plankion Research. Vol 14 (8): 1031-1054.

Magazzu, G. \& F. Decembrini, 1990. Primary Production y Picoplankton assimilation in the Ross Sea (Antártica). Nat. Sc. Com. Ant. Ocean. Camp. 1987-88. Data Rep. I: 107-157.

Margalef, R., 1958. Temporal succesion and spatial heterogeneity in phytoplankton. En: A. A. Buzzati-Traverso (ed). Mar Biol.. University of California Press, Berkeley y Los Angeles:
232-329.

Meyer, M. A. \& S. Z. El-Sayed, 1983. Grazing Of Euphausia superba Dana on natural phytoplankton populations. Polar Biol., 1:193197.

Miller, D. G. \& J. Hampton, 1989. Biology and ecology of the Antartic Krill (Euphausia superba Dana): a review. BIOMASS Scientific Series (9): $1-66$.

Miguel, E. 2000. Oceanografía y dinámica en el Estrecho de Bransfield durante el verano austral de 1999. Informe sobre las actividades científicas de la Décima Expedición Peruana a la Antártida ANTAR X. Comisión Nacional Asuntos Antárticos. CONCYTEC. Lima, Perú: 83-108.

Nuccio, C.; M. Innamorati; L. Lazzara y G. Mori, 1992. Poblaciones de fitoplancton en Bahía Terra Nova, Mar de Ross. En Oceanografía en Antártica. Gallardo, V. A,. O. Ferrati y H. Moyano (eds.) ENEA. Proyecto Antárticaltalia. Centro EULA. U. Concepción-Chile: 253-262.

Schandelmeier, L \& V., Alexander, 1981. An analysis of the influence of ice on spring phytoplankton population structure in the southeast Berin Sea. Limnol. Oceanogr. 26(5): 935-943.

Shannon, C. \& W. Weaver, 1963. The mathematical theory of communication. University of Illinois Press Urbana: $125 \mathrm{pp}$.

Utermohl, H., 1958. Zur Vervollkommnung der quantitativen phytoplankton methodik. Mitt Int. Ver. Theor. angew, Limnol., 9:1-39.

Unesco. 1978. Phytoplankton manual Monogr. Oceanogr. Methodology: 6-337.

Yamamoto,T. \& A. Taniguchi, 1993. Spring and summer phytoplankton clorophyll "a" size fractions $(>10 \mu \mathrm{m}$ and $<10 \mu \mathrm{m})$ in the of fshore waters around Japan. J. Fac. Appl. Biol. Sci. 32: I -6. 